Ebisu

Études japonaises

Études japonaises

47 | printemps-été 2012

Catastrophes du 11 mars 2011, désastre de

Fukushima : fractures et émergences

\title{
Reconstruire ses souvenirs : le nettoyage des photographies emportées par le tsunami à Kesennuma
}

Reconstructing Memories: Restoring Photographs Swept Away by the

Kesennuma Tsunami

思い出の復興一気仙沼市の津波で流された写真洗浄

\section{Coralie Castel}

\section{CpenEdition}

\section{Journals}

Édition électronique

URL : http://journals.openedition.org/ebisu/478

DOI : $10.4000 /$ ebisu.478

ISSN : 2189-1893

Éditeur :

Institut français de recherche sur le Japon (UMIFRE 19 MAEE-CNRS), Maison franco-japonaise

\section{Édition imprimée}

Date de publication : 1 juin 2012

Pagination : 229-240

ISSN : 1340-3656

Référence électronique

Coralie Castel, «Reconstruire ses souvenirs : le nettoyage des photographies emportées par le tsunami à Kesennuma », Ebisu [En ligne], 47 | printemps-été 2012, mis en ligne le 03 avril 2014, consulté le 30 avril 2019. URL : http://journals.openedition.org/ebisu/478; DOI : 10.4000/ebisu.478 


\title{
Reconstruire ses souvenirs Le nettoyage des photographies emportées par le tsunami à Kesennuma
}

\author{
Coralie CASTEL
}

«La vague n'emporte pas les souvenirs ». Telle est la phrase que répète inlassablement Takai Shinji 高井晋次, un agriculteur de Kesennuma 気仙沼. Depuis fin mars 2011, il a rassemblé quelques habitants qui reconstituent bénévolement les albums photos retrouvés dans les décombres du tsunami. Dans cette ville, les pertes humaines ont été très importantes : 2000 morts et disparus pour 73000 habitants. Parmi les décombres, les équipes de sauveteurs ont dégagé et mis de côté de grandes quantités de photographies. Jugeant qu' " il n'était pas bon de les laisser là ", des bénévoles ont alors formé le Projet de nettoyage des photographies (Shashin senjō purojekuto 写真洗浄プロジェクト) afin de les nettoyer, de les classer et de les exposer dans le but ultime de les rendre à leurs propriétaires ou à leurs familles.

La pratique et les usages ordinaires de la photographie participent à la construction de la mémoire personnelle, créent et entretiennent le lien social. L'initiative de Kesennuma illustre l'importance de ce rapport à la photographie, particulièrement fort au Japon. Sa façon de se manifester ici dans des circonstances exceptionnelles répond à ce que J. Langumier et S. Revet appellent les " dynamiques sociales et collectives » suscitées par les catastrophes (Langumier \& Revet 2011 : 80). Leur description permet de

Coralie Castel est doctorante en ethnologie au sein du Laboratoire d'ethnologie et de sociologie comparative, université Paris Ouest Nanterre La Défense. Sa thèse porte sur la mise en scène des liens sociaux par la photographie au Japon. 
saisir comment les habitants parviennent à vivre quotidiennement, sur le long terme, avec les conséquences d'un désastre encore bien présent.

C'est dans cette optique que je vais ici rendre brièvement compte d'une ethnographie du projet réalisée en août 2011. J'ai observé la réalisation du nettoyage des photographies et recueilli les discours qui entourent l'activité bénévole : cette enquête tend à montrer que la prise en charge matérielle des photographies participe en définitive au rétablissement de la société locale dans son ensemble, par une remise en ordre du monde ébranlé par le tsunami.

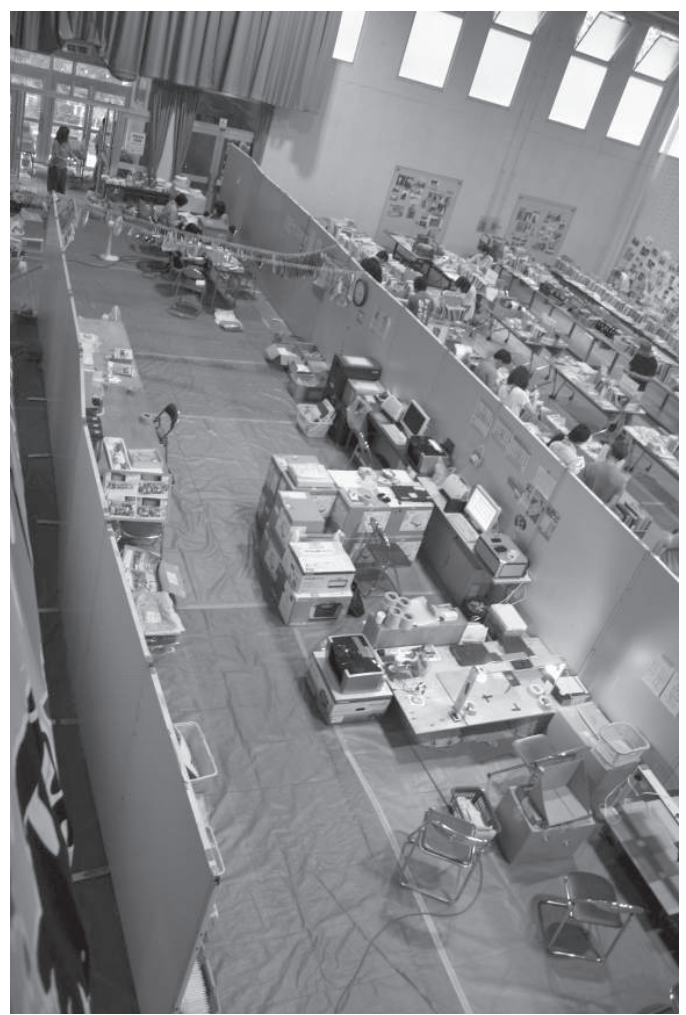

Fig. 1 


\section{Rationaliser et classer la masse des photographies}

Takai Shinji, ex-ingénieur reconverti dans la production des fraises, a vu son exploitation détruite. Deux semaines après la catastrophe, une fois le premier temps d'urgence passé, il vit toujours réfugié au centre de secours Hashikami 階上 et se demande «ce [qu'il] pourrait faire, de ce qui est là, devant [ses] yeux ». Il expose ainsi son initiative : les photographies retirées des ruines par les équipes de sauveteurs s'entassaient devant les maisons ; alors, dit-il, "j'ai eu cette idée toute simple, je voulais juste les nettoyer ».

Rapidement, les activités s'organisent sous le nom de Projet de nettoyage des photographies. Il est localisé d'abord dans l'enceinte du refuge, puis dans un gymnase d'un quartier voisin. Takai attire l'attention des medias, notamment par son blog, ce qui suscite l'arrivée de renforts de tout le pays'. Le premier jour de nettoyage, le $1^{\text {er }}$ avril, 600 photos sont restaurées. Elles sont 15000 à la fin du mois, et un million cinq mois plus tard. En août, des sacs entiers de photographies souillées continuaient d'arriver au gymnase chaque jour.

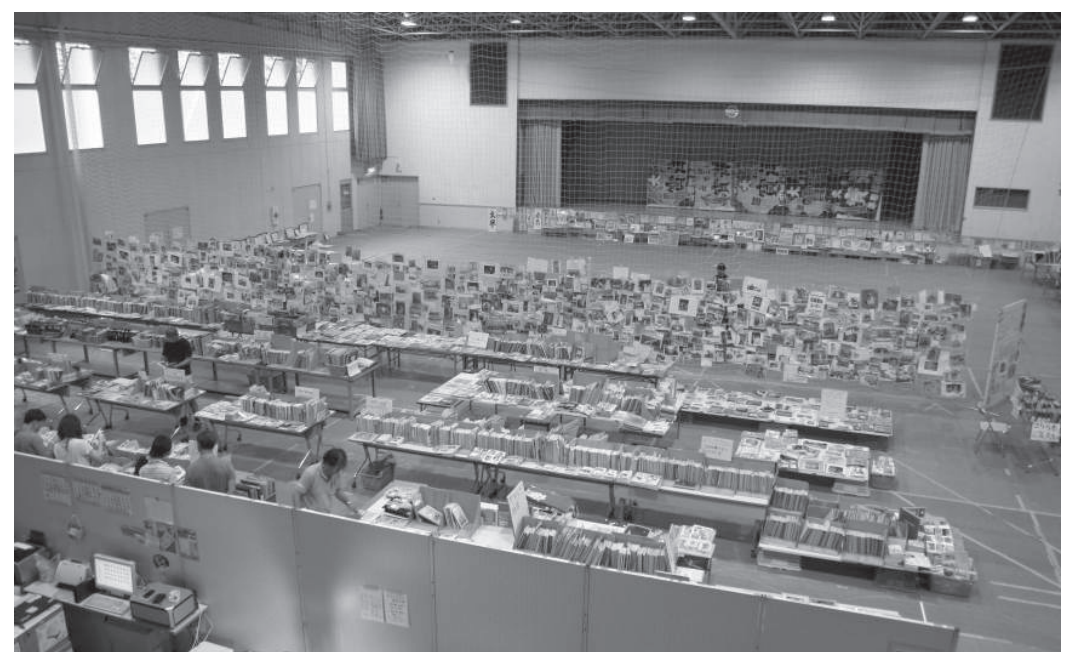

Fig. 2

1. Voir : <http://ameblo.jp/kcberry> (dernière consultation le 21/11/2011). 
Le traitement d'une telle quantité de documents nécessite évidemment une organisation rigoureuse. Dans le gymnase, des panneaux amovibles séparent la salle d'exposition de l'espace de traitement des photographies (fig. 1 et 2). À l'intérieur de celui-ci, des cartons vides délimitent les différents " postes " de travail : découpage des albums salis et lavage à l'eau des photographies ; séchoirs ; reconstitution des albums à l'aide de pochettes propres reliées ensemble par du ruban adhésif; postes informatiques de numérisation des albums "neufs " pour la constitution d'une base de données. L'espace d'exposition est quant à lui divisé entre les albums, les photographies isolées, et d'autres objets variés, rassemblés sous la dénomination d' « objets-souvenirs " (omoide no shina 思い出の品).

Les photographies sont regroupées en paquets, supposés appartenir à une même famille, qui reçoivent chacun un nom, fonction du lieu où ils ont été retrouvés, et un numéro. Ils sont notés sur une bande de ruban adhésif résistant à l'eau et repositionnable, qui les accompagne tout au long des différents stades de traitement.

Lors de la reconstitution des albums, le bénévole en charge passe en revue chaque photographie du paquet afin d'en sélectionner une qu'il juge plus représentative : portrait d'une personne dont le visage est récurrent, ou

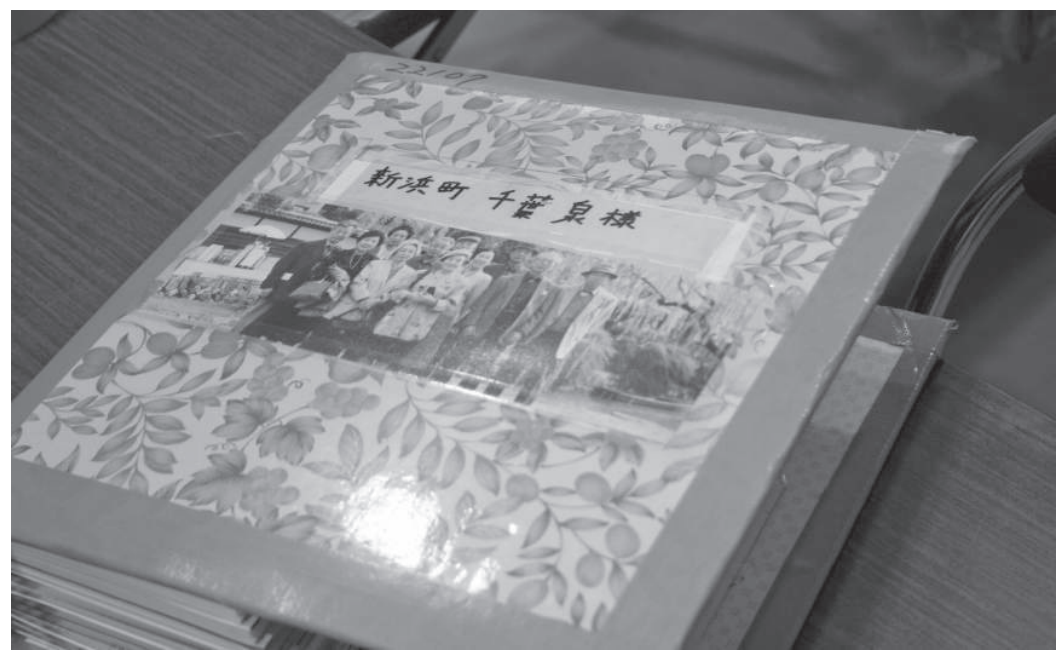

Fig. 3 
photo de groupe. Cette photographie principale est collée sur la couverture, pour que les propriétaires qui visitent le gymnase puissent éventuellement repérer plus facilement leurs propres albums (fig. 3), puis numérisée pour constituer la base de données. L'index informatique ne comporte donc que des « couvertures» d'albums, et pas leurs contenus.

Le traitement matériel des photographies passe ainsi par des étapes formellement définies et des règles strictes et méticuleuses afin d'ordonner la masse d'informations. Or, l'observation des interactions entre bénévoles montre que c'est précisément par la gestion concrète des données qu'est gérée la charge émotionnelle des photographies.

\section{L'impact émotionnel du souvenir}

Le gymnase n'expose pas que des photographies : bien que l'essentiel du temps des bénévoles soit consacré à celles-ci, ils nettoient également divers objets personnels sortis des décombres et confiés par défaut à Takai car « la ville de Kesennuma était débordée, et personne ne savait quoi en faire ". Diplômes, trophées sportifs, bijoux, cartables, carnets, mais aussi portraits

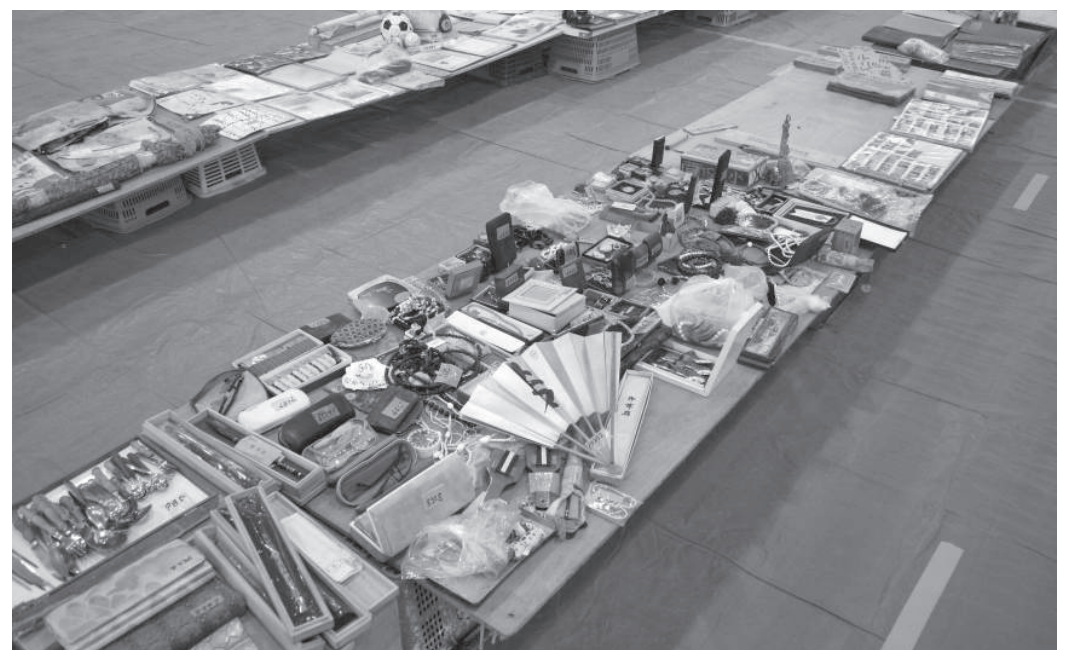

Fig. 4 
et tablettes funéraires : un inventaire hétéroclite d'articles dont le seul point commun est de correspondre à la définition vague de l'" objet-souvenir ». Le critère semble être celui de la valeur affective renfermée par les objets. Ils mettent en scène les vies passées d'avant la catastrophe, et forment les " ego-musées ", pour reprendre le terme d'A. Muxel (Muxel 1996 : 152), anonymes des victimes auxquelles ils appartiennent (fig. 4).

Dans le cas des photographies, cette valeur affective est mise en évidence par leur manipulation au cours des différents stades de nettoyage. Bien que les instructions pour le lavage à l'eau soient d'une simplicité enfantine et que les responsables répètent à l'envi que " tout le monde peut le faire ", la tâche de nettoyage semble parfois difficile. Il s'agit en effet de frotter la boue ou le sable en abîmant le moins possible les visages figurant sur les images, parfois en vain (fig. 5). En somme, il incombe au bénévole de sauvegarder une identité, la trace d'une personne dont on ne sait pas si elle est morte ou vivante. Le visionnage d'un grand nombre de photographies fait ressortir les pratiques référentielles communes (Bourdieu 1965) : photographies de mariage, portraits de nouveaux-nés, photos de groupes. Les bénévoles se les passent de mains en mains, se projettent dans ces clichés anonymes et en font le sujet principal de leurs discussions. Chacun se prend à retracer

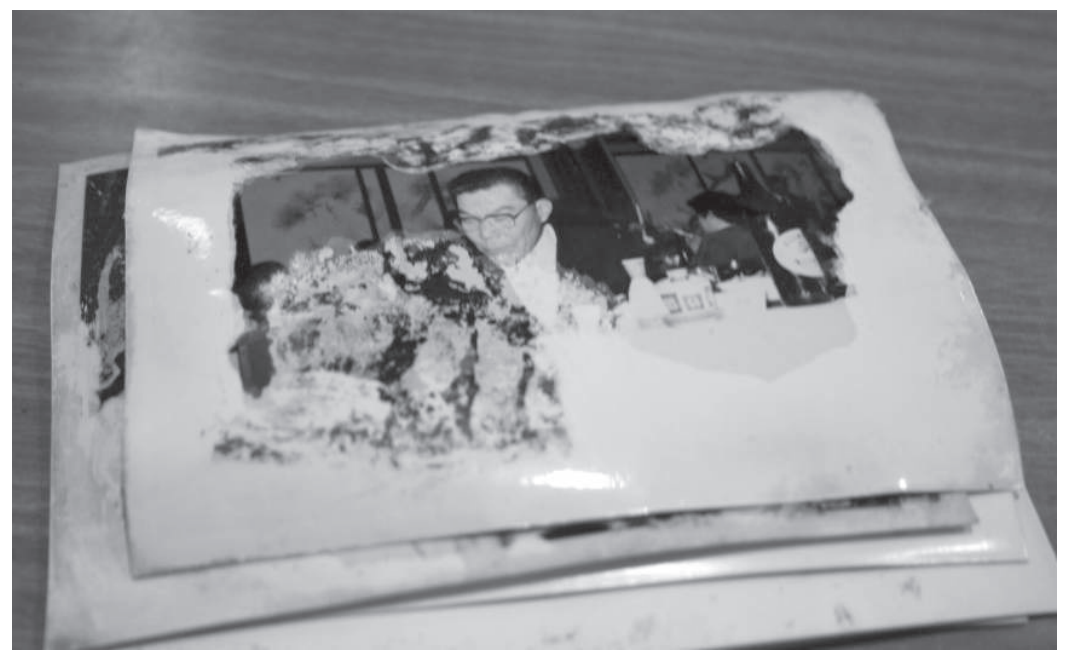

Fig. 5 
des histoires familiales à partir des événements importants qu’il voit défiler sous ses yeux, et commente une attitude amusante, ou le sourire d'un bébé. L'impact émotionnel est donc non négligeable.

Cependant, la plupart des participants mettent plutôt en avant le côté positif de leur action, pour les habitants, et aussi pour eux-mêmes. Les bénévoles apparaissent soudés et enthousiastes, mais surtout, ils répètent que ce sont les photographies elles-mêmes qui "donnent du courage ": elles forment en effet une collection de scènes idéales du bonheur familial (sur la sélection des photographies et la constitution du récit familial idéal par la fabrication d'albums : Kaufmann 2004, Favart 2001). Pour une professeure venue de Hiroshima encadrer des étudiants bénévoles, voir toutes ces photos permet de réaliser " qu'ici, tout n'est pas perdu ». Le traitement des photographies des victimes du tsunami constitue une thérapie de groupe pour ceux qui y participent, et donne lieu à l'élaboration d'un discours sur la valeur et l'importance de la photographie comme moyen de " sauvegarder les souvenirs".

Les photos irrécupérables ne rejoignent pas les albums reconstitués. Mais, d'après Takai, "d'un point de vue du ressenti, on ne peut pas les mettre à la poubelle » : elles renferment toujours, malgré leur surface effacée, un contenu affectif. Ces photographies sont envoyées à un temple de Kamakura 鎌倉, le Zuisenji 瑞泉寺. Elles sont alors détruites lors d'une crémation de photographies, rituel qui, en temps ordinaire, rassemble chaque année quelques dizaines de personnes. Le moine responsable de son organisation, rencontré sur place, explique qu'elles souhaitent " mettre en ordre leurs sentiments " et "soulager leur cœur en rendant les photos au ciel ». Les propriétaires viennent idéalement brûler eux-mêmes leurs photographies, mais cette année, du fait de la catastrophe, celles de Kesennuma ont été envoyées par la poste.

\section{Des réseaux nationaux pour un rétablissement local}

La prise en charge des photographies dépasse donc l'échelle de la ville : le soutien national, tant matériel qu'humain, a été décisif. En effet, le soutien institutionnel local est faible et insuffisant. Ainsi, la police avait commencé à se charger du traitement des tablettes funéraires mais s'est vue rapidement 
débordée par leur nombre : c'est donc le Projet de nettoyage des photographies qui en a pris le relais.

La solidarité nationale est entretenue par une forte médiatisation : de nombreux journaux et émissions de télévision ont fait des reportages dès le mois d'avril, et Takai a répondu à de nombreuses interviews. À chaque fois, il le signale sur son blog, ce qui permet d'entretenir l'écho du projet sur Internet. Grâce à cette large diffusion de l'information, des bénévoles ont afflué de tout le pays depuis le mois d'avril. En août, une vingtaine de bénévoles venus de Tokyo ou du Kansai se relayaient chaque jour. Parmi eux, certains lient connaissance et reviennent ensemble plusieurs fois à Kesennuma. Ils sont hébergés sur place dans des familles avec qui ils établissent des liens forts. Chaque voyage comporte une perspective festive : achat de cadeaux avant le départ, et grand dîner rassemblant bénévoles, famille d'hébergement élargie et voisins à leur arrivée.

L'aide au niveau national est également matérielle : ainsi les entreprises de photographie, telles Fujifilm, Kodak et Canon se sont impliquées dans le projet. Fujifilm a prodigué des conseils pour le nettoyage et la conservation des photographies et a diffusé chez tous ses photographes affiliés une note technique au cas où ils auraient à s'occuper de photographies retrouvées après le tsunami. Enfin, dans une de ses usines à Kanagawa 神奈川, elle a mis à contribution des employés et leurs familles qui ont participé chaque week-end au nettoyage de photographies envoyées par la poste. Plusieurs associations de bénévoles du Kantō 関東 ou du Kansai 関西 se font aussi envoyer des caisses de photos, qu'ils retournent ensuite par la poste, participant ainsi de chez eux au nettoyage. Kodak a fourni deux imprimantesscanners de très grande vitesse et on peut encore citer un photographe indépendant qui a offert plusieurs cadres en bois facilitant la numérisation des albums. De nombreuses universités envoient du matériel (bacs de tri, sécheuses à air chaud, albums vides, ruban adhésif professionnel...) ou des étudiants. L'association des universités chrétiennes a ainsi recruté des bénévoles spécialement pour participer au nettoyage des photographies.

L'importance attachée à la préservation des liens locaux par l'équipe bénévole se mesure aux cartes de vœux du nouvel an retrouvées sur place, véritables archives du réseau d'interconnaissance des familles, qui sont aussi nettoyées et exposées à côté des photographies. Enfin, l'ampleur du soutien 
national permet au projet d'atteindre peu à peu son but initial : rendre les photographies à leurs propriétaires ou à leurs familles. En septembre, une centaine d'habitants visitait le gymnase chaque jour. Une vingtaine retrouvait des photographies de leur famille, d'amis ou de voisins. Leur restitution resserre alors les liens locaux.

Si les données sont numérisées pour en faciliter l'accès, pour autant, elles ne seront pas à disposition sur Internet. La base de données est en réalité à utilisation locale : Takai souhaite se déplacer chez les habitants avec un disque dur, en particulier chez les personnes âgées, afin de visionner avec elles les photographies : " on se rassemble, on boit du thé... on regarde des vieilles photos et ça fait de la conversation. Même s'ils ne trouvent pas de photos à eux, on passe un bon moment " : la communication et le partage sont donc des résultats de la manipulation des photographies, qui « donnent du courage " et permettent d'" aller de l'avant ", selon l'expression maintes fois écrite dans le livre d'or, pour continuer à vivre. La conséquence ultime des actions nationales est donc de recréer et de renforcer le tissu social local.

\section{Conclusion}

Susan Sontag qualifie la photographie à la fois de "pseudo-présence " et de "marque de l'absence " (Sontag 1977 : 16). Les photographies de Kesennuma soulignent la disparition des êtres chers et des lieux d'habitation familiers en même temps qu'elles les rappellent au souvenir. À l'heure actuelle, les corps de milliers de disparus, emportés par la vague, n'ont encore pas été retrouvés. Se pose alors pour leurs proches le problème de la trace de leur existence : il ne reste rien d'eux, à part, peut-être, quelques photographies ou objets personnels. Parmi les habitants venus chercher des photographies, nombreuses sont les mères qui souhaitent retrouver des images de leurs enfants quand ils étaient petits. Même s'ils sont sains et saufs, l'absence d'indices du passé paraît causer une grande souffrance.

En définitive, le nettoyage et le classement des photographies comme opérations de sauvegarde rendent possible la remise en ordre du monde ébranlé, désorganisé par le tsunami. Dans cette table rase qu'est le paysage dévasté de Kesennuma, il s'agit de reconstruire son identité en retrouvant 
des marques de « ce qui a été », pour reprendre les mots de Barthes (Barthes 1980 : 120). La reconstruction de soi ne peut se faire sur du vide, et passe donc par le collectif, l'entraide et les échanges. Il est à cet égard significatif que les arrière-plans des photographies ou les vues aériennes de lieux soient fréquemment sujets à des échanges entre habitants et bénévoles : c'est la vie à Kesennuma qu'il s'agit de sauvegarder. Comme le dit un anonyme sur le livre d'or : «les photographies sont bien la preuve que j'ai vécu ici ».

30 novembre 2011

\section{Post-scriptum}

Si le présent article se base sur une ethnographie réalisée moins de six mois après la catastrophe, un retour sur le terrain en janvier 2012 a permis de compléter et de préciser les données d'enquête, notamment au niveau de l'institutionnalisation du Projet de nettoyage. Celui-ci a désormais intégré l'Association de reconstruction de Kesennuma (Kesennuma fukkō kyōkai 気仙沼復興協会), ce qui permet à une dizaine de membres permanents d'être rémunérés par l'État japonais pour leurs activités autour des photographies. Les développements de ce complément de terrain feront l'objet d'une analyse ultérieure, mais il est d'ores et déjà possible de dire que l'évolution du projet confirme son ancrage dans une dynamique à long terme, laquelle concerne l'ensemble de la région touchée par le tsunami. 


\section{Bibliographie}

BARTHES Roland, 1980

La Chambre claire. Note sur la

photographie, Seuil, Paris.

BOURDIEU Pierre, 1965

Un art moyen. Essai sur les usages sociaux de la photographie, Paris, Éditions de Minuit

FAVART Evelyne, 2001

«Albums de photos de famille et mémoire familiale : regards croisés de femmes de trois générations », Dialogue 154 : 89-97.

KAUFMANN Jean-Claude, 2004

L'invention de soi. Une théorie de l'identité, Paris, Armand Colin.

\section{LANGUMIER Julien}

\section{\& REVET Sandrine, 2011}

«Une ethnographie des catastrophes est-elle possible ? Coulées de boue et inondations au Venezuela et en France ", Cahiers d'anthropologie sociale, 7 : 77-90.

\section{MUXEL Anne, 1996}

Individu et mémoire familiale, Paris, Nathan.

\section{SONTAG Susan, 1977}

On Photography, London, Penguin.

\section{Bibliographie indicative sur le rapport à la photographie au Japon}

BEN-ARI Eyal, 1991

«Posing, Posturing and Photographic

Presences: a Rite of Passage in a Japanese Commuter Village », Man, 26 (1) : 87-104.

\section{CHALFEN Richard, 1987}

Snapshot Versions of Life, Bowling Green, Bowling Green State University Popular Press.

DUTEUIL-OGATA Fabienne, 2007 «La photo-interview : dialogues avec des Japonais », Ethnologie française, 37 (1) : 69-78.

PLATH David W., 1995

Durable Snapshots, Mutable Selves: or Is It Vice Versa?, Illinois, Association for Asian Studies Annual Meeting.

RIPERT Blandine, 2004

«Ritualiser l'éphémère», EspacesTemps.net : <http:// espacestemps.net/document631.html> (dernière consultation le 5/02/2012).

SAKAMOTO Ikkō 坂元一光, 1993 «Shashin no jinruigaku no tame ni » 写真の人類学のために (Pour une anthropologie de la photographie), Bulletin of Oita prefectural College of Arts and Culture 31 : 145-151, Oita Prefectural College of Art and Culture.

TSUNODA Ryūichi 角田隆一, 2002 «Kazoku shashin no shakaigakuteki 
ichi kōsatsu : "kioku" kara miru shashin jissen to sono shakaiteki kinō » 家族写 真の社会学的一考察—「記憶」からみる 写真実践とその社会的機能 (Réflexion sociologique sur la photographie de famille: pratiques et fonction sociale de la photographie vues à partir de la « mémoire »), Gendai shakai riron kenkyū 現代社会理論研究 (The Journal Studies in Contemporary Social Theory) 12:50-68.

YAMASHITA Kiyomi 山下清美, 2005 "Shashinkan de toru kazoku shashin no kachi o saguru » 写真館で撮る家族写真の 価値を探る (À la recherche de la valeur des photographies de famille prises en studio), Studio Memo 167, Tokyo, Fujifilm 富士フィルム. 